\title{
A QUIMIOTERAPIA E SEUS EFEITOSADVERSOS: RELATO DE CLIENTES ONCOLÓGICOS
}

Lenícia Cruz Soares ${ }^{1}$, Andréia Burille², Milena Hohmann Antonacci ${ }^{3}$, Maria da Glória Santana ${ }^{4}$, Eda Schwartz ${ }^{5}$

RESUMO: Trata-se de uma pesquisa qualitativa, com abordagem exploratória e descritiva, com o objetivo de conhecer como os pacientes em quimioterapia lidam com os efeitos adversos do tratamento. O cenário da pesquisa foi a Unidade de Oncologia, de um hospital da região Sul do Rio Grande do Sul. Participaram do estudo cinco pacientes em tratamento quimioterápico. A coleta de dados ocorreu em setembro de 2008, por entrevista aberta com questão norteadora. Os entrevistados relataram que apresentaram efeitos adversos ao tratamento, alguns em maior, outros em menor intensidade. Mencionaram que possuem informações superficiais sobre a quimioterapia e seus efeitos. Referiram que usam medicação para amenizá-los, sendo que alguns se automedicavam. Também destacaram a importância fé/religiosidade no tratamento. PALAVRAS-CHAVE: Enfermagem; Relações enfermeiro-paciente; Cuidados de enfermagem; Quimioterapia.

\section{CHEMOTHERAPY AND ITS ADVERSE EFFECTS: REPORT OF ONCOLOGY CLIENTS}

ABSTRACT: It is a qualitative research, with exploratory and descriptive approach, with the objective of knowing how patients in chemotherapy deal with the adverse effects of the treatment. The scenery of the research was the Oncology Unit of a hospital of the South area of Rio Grande do Sul. Five patients in chemotherapy treatment participated of the study. The collection of data happened in September of 2008, by open interview with a guideline question. The interviewees told that they presented adverse effects to the treatment, some in larger, other in smaller intensity. They mentioned that they possess superficial information about chemotherapy and their effects and they referred to use medication to soften them, and some of them referred to take medication without medical advice. They also detached the importance of faith and religiosity on the treatment.

KEYWORDS: Nursing; Nurse-patient relationships; Nursing care; Chemotherapy.

\section{LA QUIMIOTERAPIA Y SUS EFECTOS ADVERSOS: RELATO DE DE CLIENTES ONCOLÓGICOS}

RESUMEN: Se trata de una investigación cualitativa, con abordaje exploratorio y descriptivo, con el objetivo de conocer cómo los pacientes en quimioterapia lidian con los efectos adversos del tratamiento. El escenario de la investigación fue la Unidad de Oncología, de un hospital de la región sur de Rio Grande do Sul. Participaron del estudio cinco pacientes en tratamiento quimioterápico. La recolección de datos ocurrió en septiembre de 2008, a través de entrevistas abierta con cuestión orientadora. Los entrevistados informaron que presentaron efectos adversos al tratamiento, algunos en mayor, otros en menor intensidad. Mencionaran que tienen informaciones superficiales sobre la quimioterapia y sus efectos y afirmaran que utilizan medicación para aliviarlos, siendo que, algunos se automedican. También destacaron la importancia fe/religiosidad en el tratamiento.

PALABRAS CLAVE: Enfermería; Relación enfermera-paciente; Atención en enfermería; Quimioterapia.

${ }^{1}$ Enfermeira do Hospital Escola Universidade Federal de Pelotas-UFPel-FAU. Especialista em Projetos Assistenciais e Mestranda do Programa de Pós-Graduação em Enfermagem da UFPel. Membro do Núcleo de Condições Crônicas e suas Interfaces-NUCCRIN-UFPel. ${ }^{2}$ Acadêmica do Curso de Graduação em Enfermagem da Faculdade de Enfermagem da UFPel. Bolsista de Iniciação Científica. Membro do NUCCRIN

${ }^{3}$ Enfermeira graduada pela Faculdade de Enfermagem da UFPel.

${ }^{4}$ Doutora em Enfermagem. Professora Associada da Faculdade de Enfermagem e Obstetrícia da UFPel. Pesquisadora do NUCCRIN

${ }^{5}$ Doutora em Enfermagem. Docente da Faculdade de Enfermagem e Obstetrícia da UFPel. Pesquisadora do NUCCRIN

Autor correspondente:

Lenícia Cruz Soares

Universidade Federal de Pelotas

Rua General Osório, 450/103 - 96020-000 - Pelotas-RS, Brasil

Recebido: 29/10/08

E-mail: lenicia.soares@gmail.com 


\section{INTRODUÇÃO}

O câncer é considerado um dos maiores problemas de saúde pública na atualidade, sendo a segunda causa de morte em regiões mais desenvolvidas. No Brasil soma-se à gravidade da doença a ocorrência de diagnósticos tardios, ocasionando muitas vezes maior estadiamento da doença. Associado a isso, nem sempre os tratamentos iniciam logo após o diagnóstico, perdendo-se muito tempo, o qual é fator fundamental para o paciente oncológico.

A partir da prática profissional, percebe-se que os pacientes com câncer, em geral, submetem-se a tratamento que, na maioria das vezes provoca uma série de consequências físicas, emocionais e sociais. Essas mudanças requerem atenção e suporte maior por parte da família e da equipe multiprofissional. Não raro, se inicia um processo no qual vivenciam diversas perdas de autonomia no cotidiano e alterações nos hábitos de vida, com necessidade de criar novas maneiras de viver e adaptar-se à realidade apresentada, uma vez que esse paciente passa a depender de medicações e a conviver com os efeitos adversos da terapêutica.

Os agentes antineoplásicos vêm sendo amplamente utilizados no tratamento adjuvante ou paliativo de neoplasias malignas. Contudo, por ser uma forma de tratamento sistêmico, a quimioterapia atinge indiscriminadamente todas as células do organismo, principalmente células de rápida proliferação, produzindo efeitos adversos, os quais estão diretamente relacionados ao estado do paciente, estadiamento da doença e também das drogas usadas ${ }^{(1)}$.

Acredita-se que, para proporcionar assistência de enfermagem resolutiva e integral ao paciente oncológico, se torna relevante o contínuo aprimoramento dos conhecimentos técnico-científicos, bem como o estreitamento das relações interpessoais, promovendo ações de saúde e práticas educativas eficazes no decorrer do tratamento, que possibilitem minimizar o sofrimento de todos os envolvidos no processo de cuidar ${ }^{(2)}$.

Diante do exposto e a partir das reflexões das nossas vivências no cuidado ao paciente oncológico em tratamento quimioterápico, compreende-se a necessidade de ampliar os conhecimentos para melhor assisti-los, tendo em vista que muitos apresentam diversos efeitos adversos ao tratamento, em variados graus de intensidade. Assim, este estudo tem como objetivo conhecer como os pacientes em quimioterapia lidam com os efeitos adversos do tratamento.

\section{MÉTODOS}

Trata-se de pesquisa de abordagem qualitativa do tipo descritiva e exploratória, realizada no mês de setembro de 2008, em uma Unidade de Oncologia de um hospital de ensino, da região Sul do Rio Grande do Sul.

Participaram do estudo cinco pacientes em tratamento quimioterápico, que atenderam os critérios: possuir diagnóstico de câncer e estar em tratamento quimioterápico; concordar em participar do estudo e com a divulgação dos resultados deste em meios científicos

Foram seguidos os procedimentos éticos exigidos pela Resolução n. 196/96 ${ }^{(5)}$ do Conselho Nacional de Saúde do Ministério da Saúde, sendo a pesquisa aprovada pelo Comitê de Ética em Pesquisa da Faculdade de Odontologia da Universidade Federal de Pelotas sob o parecer n. 035/2008. Os sujeitos assinaram o Termo de Consentimento Livre e Esclarecido e para garantir o anonimato, foram identificados pela letra E, seguido do número da entrevista.

Para a coleta dos dados utilizou-se a entrevista aberta, com a questão norteadora: Fale sobre como o (a) senhor (a) lida com os efeitos adversos do tratamento quimioterápico. A coleta ocorreu durante duas semanas, sendo que os pacientes foram entrevistados em uma sala reservada após a sessão de quimioterapia. Podemos entender por entrevista aberta, aquela que parte de certos questionamentos básicos que interessam à pesquisa e que, a partir daí, oferece amplo campo de interrogativas, que vão surgindo à medida que se recebem as respostas do informante ${ }^{(3)}$. Desse modo, permite aos sujeitos seguir espontaneamente a linha de seu pensamento e de suas experiências dentro do foco principal colocado pelo investigador e a participar na elaboração do conteúdo da pesquisa ${ }^{(4)}$.

Para a organização e análise dos dados, utilizouse a análise temática ${ }^{(6)}$. Assim, obtiveram-se três temas: Reações adversas da quimioterapia; Orientações recebidas; Estratégias utilizadas para lidar com os efeitos adversos.

\section{RESULTADOS E DISCUSSÕES}

\section{Apresentando os sujeitos}

O Sujeito E1: sexo feminino, 67 anos, ensino fundamental incompleto, é do lar, tem diagnóstico de carcinoma de mama e está há quatro meses em 
quimioterapia; E2: sexo feminino, 60 anos, ensino fundamental completo, é aposentada, tem como diagnóstico neoplasia maligna de ovário e está há sete anos em quimioterapia; E3: sexo feminino, 54 anos, ensino fundamental incompleto, é empregada doméstica, possui diagnóstico de carcinoma de mama e faz quimioterapia há quatro meses; E4: sexo feminino, 49 anos, ensino superior completo, professora, tem diagnóstico de adenocarcinoma de cólon e faz quimioterapia há cinco meses; E5: sexo masculino, 22 anos, ensino fundamental completo, é pecuarista, possui diagnóstico de carcinoma de testículo e está há dois anos em quimioterapia.

\section{Reações adversas da quimioterapia}

Os entrevistados referiram efeitos adversos ao tratamento antineoplásico, alguns em maior, outros em menor intensidade:

Fico cansada [...] parece que eu tenho um peso no corpo. Tenho muita vontade de ficar só deitada, e dói os braços, a perna, dói tudo (E1).

Era uma dor queimada, parecia que tinha fogo dentro de mim. Foi horrível, horrível. Eu pedia até prá morrer, que eu não resistia aquela dor [...]. Tudo que caía no meu estômago voltava e me deu diarréia, me deu dor no estômago. Eu não podia sentir o cheiro da comida. (E3).

Eu tive vômito, eu vomitei, eu tive diarreia forte (E4).

Os efeitos adversos são considerados uma das principais limitações do tratamento, podendo apresentarse de forma mais severa ou mais amena. Dentre eles pode-se citar: mielossupressão, cicatrização deficiente de feridas, alopécia, lesão no epitélio gastrintestinal, esterilidade, teratogenicidade, entre outros ${ }^{(1)}$.

Observa-se, nesta pesquisa que as náuseas e os vômitos são os efeitos colaterais mais frequentes nos entrevistados, causando-lhes um desgaste grande, tanto no que se refere ao aspecto fisiológico como emocional.

Os efeitos tóxicos da quimioterapia podem se evidenciar imediatamente, como dor após administração intravenosa, ou mais tarde, como a alopécia. Também, muitos pacientes com câncer não recebem tratamento adequado para dor, apesar de ser um problema simples de ser resolvido ${ }^{(7)}$.

O tratamento quimioterápico pode causar danos de natureza psicossocial e problemas de autoimagem que são provenientes da alopécia, das disfunções sexuais, da ansiedade e o medo:

Você não sente vontade de nada [...]. Esse remédio a gente sente na gente como ele é forte, ele dá um abalo, mesmo que a gente não queira, ele te dá, você fica muito mais sensível, tem mais medo (E4).

A enfermidade e o tratamento geram estresse e angústia emocional, pois trazem consigo medo de morrer, abandono de planos para o futuro, mudanças físicas, psíquicas e sociais. Ter a doença e/ou estar em tratamento significa estar constantemente convivendo com incertezas ${ }^{(8)}$.

Embora possa causar inúmeros efeitos adversos, a quimioterapia representa para esses pacientes uma nova chance, uma oportunidade de recuperar a saúde. As adversidades e as limitações se tornam pequenas, frente à força de vontade desses pacientes.

\section{Orientações recebidas}

Constata-se que os entrevistados possuíam informações superficiais sobre a quimioterapia e seus efeitos, ignorando quase que totalmente a forma de ação dos antineoplásicos e os cuidados necessários relativos ao tratamento:

Só o doutor me disse que eu não comesse muito, procurasse comer em mais horários e menos. [...] disse que eu tenho que tomar bastante água (E2).

Ele não me disse nada. Quando eu me tratei, ele me disse por cima que iria cair meus cabelos, coisa assim. Essa quimioterapia que eu fiz eu não sabia o que era (E3).

A partir das falas, se pode concluir que os pacientes necessitam de mais esclarecimentos sobre as informações que recebem seguidas do diagnóstico de câncer. Também é papel da enfermagem esclarecer dúvidas decorrentes do tratamento oncológico, o que pode contribuir para reduzir a apreensão por parte do paciente, bem como torná-lo mais confiante quanto ao seu processo de $\operatorname{cura}^{(9)}$.

Nesse sentido, o maior contato e a disponibilidade para fornecer informações contam a favor do serviço no atendimento ao paciente, uma vez que, ao ampliar o conhecimento dos indivíduos, se pode 
contribuir para a melhora na comunicação entre o ele e os profissionais, promovendo maior segurança durante o tratamento.

Compete ao enfermeiro facilitar o processo de tratamento do paciente, proporcionando qualidade na assistência e diminuindo o impacto gerado nessa situação de estresse, através de orientações apropriadas acerca da terapêutica proposta ${ }^{(10)}$, como também verificar quais as estratégias utilizadas por ele, para lidar com as limitações da doença, a fim de estabelecer um plano de cuidados integral e individual, que possibilite reconhecer os principais fatores psicológicos, sociais, físicos e espirituais que afetam a sua saúde e seu bem-estar.

\section{Estratégias utilizadas para lidar com os efeitos adversos}

Os sujeitos referiram que fazem uso de medicação para amenizar os efeitos adversos apresentados. Nas falas seguintes observa-se a prática da automedicação e, por outro lado, que nem sempre a medicação prescrita promove o efeito desejado:

Só prá dor que eu tomei Tylenol, por minha conta. Tomei três tylenol de uma vez só [...]. Para vômito eu tomei aqueles comprimidinhos, que é para vômito na quimioterapia. Esses comprimidos foi o médico que deu para guria que se trata aqui, aí ela passou para mim porque sobrou remédio. [...] Quando está demais eu tomo um voltaren (E3).

Com imosec e plasil aí é bem melhor. Foi questão de dois, três dias e tava tudo beleza [...]. Às vezes um biscoito d'água me fazia mal, aí a doutora me receitou omeprazol, aí mesma coisa que tirar com a mão, muito bom (E4).

Prá amenizar os vômitos eu usei o nausedron, só que aquilo eu acho a mesma coisa que nada, quando eu não tomo é a mesma coisa quando tomo. Plasil também não adiantou [...] nenhum resolveu, sempre vomito igual (E5).

O uso de antieméticos para prevenção e manejo das náuseas e vômitos é importante, pois estes efeitos constituem obstáculo intrínseco à adesão do paciente ao tratamento e que devem ser combatidos com medicações prescritas ${ }^{(11)}$. Faz-se necessário orientar de maneira adequada, o paciente sobre diversos aspectos, principalmente sobre o tratamento quimioterápico e o de apoio, considerando que o indivíduo é responsável por sua própria saúde e pelo seu comportamento.

Ao se sentirem acometidas por alguma doença, as pessoas de modo geral ficam mais reflexivas e questionam suas próprias crenças religiosas e espirituais. Assim, os sujeitos destacaram a importância da fé/religiosidade como uma contribuição, um suporte ao tratamento, auxiliando-os no enfrentamento da doença e terapêutica:

Se eu estou aqui eu digo que é a mão de Deus que está sobre mim, que se não fosse eu não estava mais aqui não [...]. Eu busco muito a Deus, sabe, eu creio muito em Deus (E3).

Você tem que acreditar. Se você não tiver uma fé, uma crença, uma religiosidade que proteja, que você pode ficar segura, aí a gente caí mesmo. [...] Mas eu acho uma coisa muito importante ter fé e ter certeza que nada, nada depende da gente (E4).

A fé é muitas vezes vista como um "remédio" muito poderoso para os clientes com câncer, pois estes mantêm a esperança em busca de um significado e de confiança em um ser superior. Isto propicia fortalecimento e serenidade para aceitar a doença ${ }^{(12)}$. A religiosidade propicia serenidade para enfrentar as adversidades da doença, se constituindo estratégia de suporte espiritual usado frequentemente entre os pacientes com doença maligna ${ }^{(13)}$.

A convicção religiosa representa para a família e para o paciente apoio e suporte, pela qual buscam, muitas vezes, o alívio do sofrimento por que passam e depositam expectativas de milagres, de cura da doença. A religiosidade e o uso de terapias alternativas podem ser efetivas no tratamento, mas é importante realizar o tratamento medicamentoso corretamente ${ }^{(14)}$.

Muitos pacientes oncológicos, na esperança de cura através do tratamento alopático, enfrentam vários efeitos adversos desagradáveis, como a queda dos cabelos, náuseas e vômitos, entre outros. Por essa razão, acabam buscando a associação de outras medidas terapêuticas, a fim de minimizar os efeitos adversos do tratamento convencional ${ }^{(15)}$.

Nas afirmativas a seguir, se pode inferir algumas das formas encontradas para adaptarem-se aos efeitos adversos do tratamento quimioterápico, como forma individualizada e subjetiva de enfrentamento dos 
mesmos, dentre as quais se destaca o cuidado com a alimentação:

Eu tomo bastante água quando fico cansada, como bastante alface, tomate. [...] Faço força para não deitar, eu não quero me entregar, mas no fim eu tenho que deitar um pouquinho (E1).

Eu não como nada pesado, nem fritura, nem gordura, nem na semana que eu estou fazendo $e$ nem na após que eu estou fazendo. E não tomo nada quente. Quanto mais gelado eu comer, melhor é. [...] Claro que a receita eu tomo menos. Eu penso assim que quanto menos remédio eu tomar vai ser melhor prá mim. [...] Auxílio psicológico, para não cair numa crise depressiva, porque eu quero me ajudar no tratamento (E4).

A inapetência se apresenta como um problema para o paciente acometido pelo câncer e está relacionada a diversos fatores: alterações metabólicas e hormonais, alterações do paladar e olfato, presença de dor, aversão à comida, náuseas e vômitos decorrentes do próprio tratamento quimioterápico, além de fatores psicológicos como ansiedade, medo e depressão ${ }^{(1)}$.

Assim, faz-se necessário uma atenção redobrada na manutenção adequada da ingesta de alimentos, mostrando-se fundamental não só como atendimento integral no autocuidado, mas também, a fim de proporcionar os substratos necessários ao processo de recuperação ${ }^{(16)}$.

\section{CONSIDERAÇÕES FINAIS}

Apesar dos avanços tecnológicos, que possibilitaram desenvolver novas técnicas de tratamento do câncer, a doença continua representando um desafio, o qual requer medidas de enfrentamento e, também, formas para minimizar os efeitos adversos desencadeados pela terapêutica.

Neste estudo, verificou-se que os efeitos adversos da quimioterapia se manifestaram em todos os entrevistados, afetando a qualidade de vida. Evidenciouse, também, que os pacientes, ao se depararem com a doença e com as consequências do tratamento, buscam desenvolver estratégias, que vão desde alteração da alimentação até a busca pelo suporte da religiosidade/ espiritualidade para melhor enfrentá-las.

Com relação às informações sobre o tratamento quimioterápico, cuidados e efeitos adversos, observou- se que os pacientes possuíam carência significativa de informações. Dessa forma, pontua-se a necessidade dos profissionais de saúde oferecer orientações mais efetivas, com intuito de proporcionar maior segurança e compreensão sobre o tratamento, fazendo com que este seja menos temido e tenha maior possibilidade de adesão.

Considera-se relevante maior valorização das experiências de vida de cada indivíduo, por parte da equipe multiprofissional, de modo a respeitar as diferentes crenças e práticas frente às manifestações e percepções referidas pelos pacientes sobre a doença. Frente a estes aspectos, coloca-se a importância de se conhecer as estratégias utilizadas pelos clientes oncológicos no decorrer do adoecer, pois assim, pode-se direcionar com maior efetividade o olhar e as formas de cuidar.

\section{REFERÊNCIAS}

1. Bonassa EMA. Enfermagem em quimioterapia. São Paulo: Atheneu; 1996.

2. Stumm EMF, Leite MT, Maschio G. Vivências de uma equipe de enfermagem no cuidado a pacientes com câncer. Cogitare Enferm. 2008 Jan/Mar;13(1):75-82.

3. Trentini M, Paim L. Pesquisa em enfermagem: uma modalidade convergente assistencial. Florianópolis: UFSC; 1999.

4. Triviños ANS. Introdução à pesquisa em ciências sociais: a pesquisa qualitativa em educação. São Paulo: Atlas; 1995.

5. Ministério da Saúde (BR). Conselho Nacional de saúde. Diretrizes e normas regulamentadoras de pesquisa envolvendo seres humanos. Resolução n. ${ }^{\circ}$ 196, de 10 de outubro de 1996. Brasília; 1996.

6. Minayo MCS. O desafio do conhecimento: pesquisa qualitativa em saúde. 10ª ed. São Paulo: Hucitec; 2007.

7. Love RR. Complicações e Terapias de Apoio. São Paulo: Springer-Verlag, 1998.

8. Trincaus MR, Côrrea AK. A dualidade vida-morte na vivência dos pacientes com metástase. Rev Esc Enferm USP. 2007;41(1):44-51. [periódico na Internet]. [acesso em 2008 Set 10]. Disponível: http://www.scielo.br/pdf/ reeusp/v41n1/v41n1a05.pdf

9. Fernandes AFC, Rodrigues MSP, Cavalcanti PP. O comportamento da mulher mastectomizada frente às atividades grupais. Rev Bras Enferm. 2004;57(1):31-4. 
10. Leite KC, Mateus L. Enfermagem no ambulatório de um serviço de radioterapia. Nursing. 2001;(42):26-30.

11. Gonçalves JA. Controlo de sintomas no cancro avançado. Lisboa: Fundação Calouste Gulbenkian; 2002.

12. Teixeira JJV, Lefèvre F. Significado da intervenção médica e da fé religiosa para o paciente idoso com câncer. Cienc Saúde Col. 2008;13(4):1247-56.

13. Linardi AG, Dantas FA, Silva RM. Mulheres submetidas a tratamento para câncer de colo uterino: percepção de como enfrentam a realidade. Rev Bras Cancerol. 2002;48(4):493-8.

14. Servantes FL. A família do portador frente ao câncer. Terapia ocupacional: pesquisa e atuação em oncologia. Campo Grande: UCDB; 2002.

15. Jaconodino CB, Amestoy SC, Thofehrn MB. A utilização de terapias alternativas por pacientes em tratamento quimioterápico. Cogitare Enferm. $2008 \mathrm{Jan} /$ Mar;13(1):61-6.

16. Silva LMG. Breve reflexão sobre autocuidado no planejamento de alta hospitalar pós-transplante de medula óssea (TMO): relato de caso. Rev Latino-Am Enferm. 2001 Jul;9(4):75-82. 